\title{
A CNN-based Lower Limb Motion Quality Evaluation Method for Home-based Stroke Rehabilitation
}

\section{Pin Ge}

Shantou University

\section{Ziyang Huang}

Shantou University

\section{Guoliang Tang}

Shantou University

\section{Akshay Kumar}

Shantou University

\section{Seeahmed Mahmoud}

Shantou University

Qiang Fang ( $\square$ qiangfang@stu.edu.cn )

Shantou University

\section{Jian Chen}

The First Affiliated Hospital of Shantou University Medical College

\section{Research Article}

Keywords: home-based stroke rehabilitation, training quality evaluation, CNN, early supported discharge

Posted Date: June 8th, 2022

DOI: https://doi.org/10.21203/rs.3.rs-1299284/v2

License: (c) (1) This work is licensed under a Creative Commons Attribution 4.0 International License.

Read Full License 


\title{
RESEARCH
}

\section{A CNN-based Lower Limb Motion Quality Evaluation Method for Home-based Stroke Rehabilitation}

\author{
Pin $\mathrm{Ge}^{1}$, Ziyang Huang ${ }^{1}$, Guoliang Tang ${ }^{1}$, Akshay Kumar $^{1}$, Seeahmed Mahmoud ${ }^{1}$, Qiang Fang ${ }^{*}$ \\ and Jian Chen ${ }^{2 *}$
}

\footnotetext{
${ }^{*}$ Correspondence:

qiangfang@stu.edu.cn;

drchen@stu.edu.cn

${ }^{1}$ Department of Biomedical

Engineering, Shantou University,

Shantou, China

${ }^{2}$ The First Affiliated Hospital of Shantou University Medical

College, Shantou, China

Full list of author information is

available at the end of the article
}

\begin{abstract}
Background: With the rapid increase of stroke incidence in recent years worldwide, home-based rehabilitation training has become more needed, especially for remote regions or in developing countries where rehabilitation resources are scarce. Studies have demonstrated that home-based rehabilitation for poststroke patients is essential for reducing the cost as well as for providing efficient rehabilitation. Nevertheless, home-based rehabilitation training requires effective professional support and timely evaluation.

Method: In this paper, a home-based rehabilitation quality evaluation method for lower limb training was proposed. The kinematic data of a patient's lower limb during a set of selected training exercises was captured by a wireless body area sensor network (WBASN). The data was then processed by a convolutional neural network (CNN) based algorithm to classify the rehabilitation training type and to evaluate the training quality. A series of kinematic features were selected for rehabilitation quality scoring. The experiments have been conducted using 26 human participants, including 6 healthy participants and 20 stroke patients at different Brunnstrom recovery stages.
\end{abstract}

Results: An accuracy of $95.3 \%$ has been achieved for recognizing the rehabilitation training types and a statistically significant linear positive correlation $\left(R^{2}=0.9962\right)$ has been obtained between the objective scores and the Brunnstrom stages evaluated by the clinicians.

Keywords: home-based stroke rehabilitation; training quality evaluation; CNN; early supported discharge

\section{Introduction}

Cerebral stroke is an acute cerebrovascular disease, which refers to the sudden rupture of blood vessels in the brain or damage to brain tissue caused by blood vessel blockage. It has the characteristics of high morbidity, high mortality, high disability rate, high recurrence rate and many complications [1]. With the advanced acute stroke treatments, especially the medical care provided for acute stroke, there has been a rise in the overall survival rate of stroke. Nevertheless, for stroke survivors, the majority of them suffer from different forms of disability [2]. Hemiplegia is a common limb dysfunction after stroke. Relevant studies have shown that approximately $70-80 \%$ of stroke patients have various degrees of limb dysfunction [3]. However, post-stroke patients can still recover their body functionalities to a certain extent 
through appropriate stroke rehabilitation, thereby improving their quality of daily life $[2,4]$. During the rehabilitation process, the frequency, environment and training method can greatly affect the efficiency of the rehabilitation process. Therefore, professional support under supervision and nursing is critical for patients to restore their physical functionalities and reduce their dependence on others in daily life activities (DLA). Classification of impairment levels is another essential role in rehabilitation, with its manifestation of recovery extent during rehabilitation process. Brunnstrom recovery stage is a well-known classification approach that provides a standardized pattern for the recovery progress. With the simplicity of this approach, the rehabilitation progress can be divided into six Brunnstrom stages. Beginning from the stage of flaccidity to the stage at which spasticity disappears completely, the Brunnstrom stage increases from stage I to VI accordingly [5]. At present, the Brunnstrom stage classification can only be performed by experienced physicians, thus the accuracy of classification results is subject to physicians' observation inevitably. On top of that, as a long-term process, poststroke rehabilitation usually involves a large consumption of health service resources from the healthcare system $[6]$.

Early supported discharge (ESD) is a home-based rehabilitation modality that can effectively alleviate the enormous stress of health service resources. Compared to conventional medical care, the ESD rehabilitation service can lessen the hospitalization duration of patients by 8 days [7]. However, a support mechanism is required to facilitate the implementation of home-based rehabilitation for patients and their families [2]. The main components of this mechanism are automatic recognition of rehabilitation training movements and quality assessment of rehabilitation, which contribute to remote monitoring and evaluation of home-based rehabilitation training programs [4].

With the advancement in telemedicine technology and rehabilitation robot technology, more studies have been carried out on remote guidance and monitoring of home-based rehabilitation for stroke patients by using a body sensor network composed of multiple sensor nodes [8, 9]. In [10], two wireless inertial measurement units (IMU) and one wireless sEMG sensor were utilized to measure the acceleration of specified lower limb movements and EMG signals of related muscles of stroke patients. Their research used the support vector machine (SVM) classifier to classify the impairment level of lower extremities into Brunnstrom stages, and ultimately achieved an accuracy rate of $95.3 \%$. However, the type of rehabilitation motions is limited in their research, with only one rehabilitation motion taken into account. In [11], 14 IMU sensors were used to develop a quality assessment system for daily rehabilitation activities. Their system can provide effective monitoring for rehabilitation activities of stroke patients, whereas due to the complex system setup, it is not suited to apply on a large scale. Liao et al. proposed a framework of rehabilitation training evaluation based on deep learning [12]. The optical motion capture system was used to acquire rehabilitation training data of stroke patients. Principal component analysis (PCA) was introduced for feature dimensionality reduction and deep learning was applied for rehabilitation training quality assessment. Nevertheless, their system was expensive, so the cost of large-scale applications was high. Therefore, there was still limited research conducted on monitoring and evaluation of quality of rehabilitation movements of lower limbs for stroke patients. 
In this paper, an innovative evaluation method based on a convolution neural network (CNN) algorithm was proposed for home-based lower-limb rehabilitation for stroke patients. A wearable body area sensor network comprising two IMU acceleration sensors was deployed for acquiring kinematic data. The convolutional neural network (CNN) algorithm was utilized to process kinematic data and to classify the rehabilitation movement types. A quality scoring system was proposed by employing a set of selected evaluation parameters to provide quantitative assessment for the lower-limb mobility of stroke patients. The proposed system contrives to monitor rehabilitation motions and evaluate the quality of home-based rehabilitation training, in particular the lower-limb training, therefore providing the essential support for the stroke patients to carry out home-based rehabilitation training.

\section{Results}

In this section, the results of the training motion classification and the rehabilitation quality scoring method will be presented followed by a discussion.

\section{Training movement Recognition Results}

Table 1 shows the training movement accuracy of the CNN-based algorithm using the $\mathrm{K}$-fold cross-validation method, where $\mathrm{K}$ is set as 5 . It can be seen that the recognition accuracy of lower limb rehabilitation movements is high, with the highest, the lowest reaching 100\%,91.7\%, respectively. Among five folds, accuracies of two folds (Split 1 and Split 2) reaches 100\%, which indicates that the training motions were all accurately predicted in these two folds. This reveals that the CNNbased model performs well in recognition of the rehabilitation training movements. In addition, A mean accuracy of $95.3 \%$ with a standard deviation of $3.9 \%$ has been obtained. Generally, this accuracy suggests a high robustness and adaptability of the model in training motion recognition of patients at different Brunnstrom stages.

\section{Quality evaluation of lower limb rehabilitation training}

Fig. 1 shows the motion acceleration curves of four rehabilitation movements for a patient at Brunnstrom stage III recorded using the IMU sensor on the thigh. The significant points have been marked in Fig. 1. These points are either the maximum or minimum points along the motion curve, which are set as the starting, ending, or turning points of the motion as it is convenient to use 2 adjacent maximum or minimum points to identify the motion cycle.

The following values of evaluation parameters can be obtained for the scoring of bridge movement: (1) the mean values of motion acceleration of starting points and turning points; (2) mean values of data sequence length of sampling points from the beginning point to the turning point and that from the turning point to the ending point. Likewise, these values can also be obtained for the remaining three rehabilitation movements. Table 2 shows the individual calculation process of each parameter and the gross score of each rehabilitation movement for a Brunnstrom stage III patient. The results of patients at other Brunnstrom stages can also be obtained through the same calculation process.

For quality evaluation, 20 patients were used for calculation, while the 6 healthy subjects were set as templates. Aeeording to Eq. 6 , the seores of healthy subjects 
wore set to 100 as the metric to which the performanee done by strolke patients are compared. Table 3 shows the mean and standard deviation of each rehabilitation exercise score for patients at different Brunnstrom stages in a quantitative scale, calculated using the proposed evaluation method. As can be observed, it is statistically significant that the scores are correlated with the Brunnstrom stage classification using Spearman's rank-order correlation method ( $p<0.05$ was used as the significance level). The scores of hip extension and knee extension are lower than those of the other three motions, whereas, for healthy participants, the scores remain the same as those of other motions. For 20 patients with different Brunnstrom stages, the mean values of the rehabilitation quality score of the 4 selected lowerlimb rehabilitation movements were calculated using the assessment method, and then were ranked according to Brunnstrom stages from low to high, as shown in Fig. 2a. In addition to strong correlation, Fig. 2a intuitively demonstrates that the scores of the proposed quality evaluation method are positively correlated with the Brunnstrom stage classification evaluated by experienced physicians, as the figure shows an upward trend of the scores from Brunnstrom stage III to VI. Spifiedly, the seores of hip extension and knee extension are lower eompared to these of the other three motions, whereas for healthy volunteers, the scores remain identical to those of other motions. Furthermore, Fig. $2 \mathrm{~b}$ shows an $R^{2}$ value of 0.9962 , which suggests a significant linear correlation exist between the mean evaluation scores and the Brunnstrom stages.

\section{Discussion}

For training motion recognition, the dimension of the applied convolutional kernel is $3 \times 3$. A $3 \times 3$ convolution kernel is considered to be much smaller than the input $9 \times 9$ feature matrix since the convolution kernel only connects with the weights of local input features, which increases the sparse interaction. Moreover, the parameter sharing mechanism of the convolution kernel reduces the number of parameters that need to be learned, and thus simplified the model and diminished the overfitting problem. In addition, the output of 32 channels of the first layer convolution can enable the network to learn more rehabilitation motion features and enhance the learning ability of the model. The batch normalization after convolution of each layer restricts the distribution of training set samples so that the model can have a better performance in learning the data distribution of training set samples, thereby reducing the deviation of training set samples, enhancing the generalization ability of the model, and improving the model classification rate of lower limb rehabilitation movement. Although the accuracy achieved was high, there are still a significantly large number of error events after iterations. The error in the recognition of rehabilitation movements can be explained as follows: (1) due to the lack of balance ability, the quality of completing the movement is low; (2) irregular error arises from the incorrect wearing and placements of IMU sensors; (3) the number of data samples is insufficient, and the generalization ability of the model needs further improvements.

On the other hand, the possible explanation is that for in-home daily life, patients have more opportunities to sit, stand, walk and turn over than to extend hip and knee. In conclusion, a patient with higher Brunnstrom stage will have a higher 
score, and have a better performance in the in-home lower limb rehabilitation. The proposed evaluation method can reflect the quality of completing lower-limb rehabilitation movements for patients at different Brunnstrom stages, thereby providing a feasible approach to objectively assess the quality of lower-limb motion for poststroke rehabilitation.

\section{Conclusion}

In order to monitor and evaluate the home-based lower limb rehabilitation training for stroke patients objectively and automatically, this paper proposed an innovative CNN-based method to recognize the type of rehabilitation training movements and evaluate the quality of the rehabilitation. Two IMU acceleration sensors were used to record the acceleration signals of four different types of lower-limb training motions, and 73-feature vectors representing the lower limb rehabilitation movement were extracted. A convolution neural network (CNN) based algorithm was utilized to automatically recognize rehabilitation movements. With the kinematic data collected from 20 patients and 6 healthy participants, the proposed recognition model reported an accuracy rate of $95.3 \%$ for the recognition of rehabilitation movements using 5 -fold cross-validation. For quality evaluation, statistically significant linear correlation has been observed between the scores of the proposed assessment method and the Brunnstrom stage classification evaluated by professional therapists. This strong correlation has validated the accuracy of the model for the quality assessment of a rehabilitation movement. The proposed method managed to monitor and provide objective quality evaluation for lower limb rehabilitation training, thereby providing feasible technical support for the implementation of ESD rehabilitation services for stroke patients. In addition to this, it also provides valuable references as well as feedback for the patients and the rehabilitation supporting teams. In the future study, the analysis of joints and relevant muscles on ankle and instep will be included, which could make the lower-limb rehabilitation for stroke patients more comprehensive and effective. In addition, an experiment with a larger number of stroke patients needs to be conducted to enhance the reliability and the efficacy of the proposed evaluation method.

\section{Method}

\section{Experimental Protocol}

Impaired lower limb motor function can lead to hemiplegia and disorders in balance and gait for stroke patients [13]. Based on the rehabilitation training that experienced rehabilitation therapists provide to stroke patients during hospitalization, combined with in-home daily activity demands of stroke patients, four rehabilitation training movements of the lower extremity were selected for the experiment, as shown in Table 4 .

The applied wireless body sensor network (WBSN) system is composed of three modules: sensor module, control module, and data transmission module. The sensor module contains two IMU sensors, each equipped with an ADXL345 accelerometer (Analog Devices, Inc., USA). It is a MEMS-based technology with the advantages of being compact, lightweight, low power consumption, programmable range, and high resolution. The sampling frequency of it is set to $40 \mathrm{~Hz}$. The control module used 
a CC2530 microcontroller (Texas Instruments, USA) with ZigBee wireless transmission. The data transmission module mainly contains the CC2530 chip, which transmits the captured data to a computer client in real-time.

Two IMU units were worn on the designated parts of a patient's lower limbs to obtain the kinematic signals during the rehabilitation motion. As shown in Fig. 3 , for the Bridge motion, the IMU sensors were worn on the middle thigh and the lower abdomen; for the other three rehabilitation motions, the sensors were worn on the middle thigh and the middle calf. For hemiplegic subjects, the sensors must be worn on the paralyzed limb, while for healthy subjects, either side can be used since the mobility of both limbs is basically the same. The actual acceleration recorded by the sensors is the addition of the gravitational acceleration and the kinematic acceleration caused by the voluntary movement of the limbs. The overall acceleration can always be projected into $\mathrm{X}, \mathrm{Y}, \mathrm{Z}$ axes to form 3 components which vary depending on different positions along the motion trajectory. These 3 components (3 variables) were used to extract features for further analysis.

In this experiment, 26 subjects were recruited, including 20 stroke patients and 6 healthy subjects. Eight of the stroke patients (inpatients) were from the Rehabilitation Medical Center of Jiaxing 2nd Hospital in Zhejiang, China, while the other twelve (outpatients) were from Longhu Hospital affiliated with Shantou University in Guangdong, China. The six healthy volunteers were from the Department of Biomedical Engineering at Shantou University, China.

Selection criteria for post-stroke patients were: 1) the patient must have no hemodynamic instability; 2) no severe cognitive impairments; 3) no dementia; 4) no major post-stroke complication; 5) stroke event happened within three months; 6) motor impairment of Brunnstrom stage III or above. Criterion 6 is included because patients at Brunnstrom stage I and II are unable to perform unsupported voluntary limb movement that the IMU sensor can capture, as required in this experiment. The motor function of Brunnstrom stage VI patients is considered near normal; therefore, the six healthy volunteers were treated as patients at stage VI [14].

The basic information of the participants is shown in Table 5. Under the guidance of rehabilitation therapists, all participants were trained on the four selected rehabilitation actions before data collection to ensure effective execution of training exercises. During the experiment, participants were asked to observe and follow the pre-recorded video of rehabilitation exercises performed by healthy individuals and maintain the same exercise speed as in the video as much as possible. Each rehabilitation exercise was repeated at least ten times per patient, depending on their motor impairment level.

The flow chart of the whole process is shown in Fig. 4. The rehabilitation movements were tracked using a body sensor network based on two inertial measurement units (IMU). The kinematic data of participants' movements, while performing rehabilitation exercises, were collected by the two IMU sensors and transmitted to the computer client via a ZigBee for further analysis. As the IMU rehabilitation data were sometimes contaminated by noise due to patients' clothes friction and wireless channel noise [15], a 9-point median filtering of $\mathrm{X}, \mathrm{Y}$ and $\mathrm{Z}$ acceleration data were applied to ensure the accuracy and reliability of the collected data and to eliminate high-frequency noise [16]. Then the pre-processed data were used for rehabilitation 
motion recognition and evaluation of quality of lower-limb rehabilitation. For motion recognition, the data went through the feature extraction, and then fed into the CNN algorithm to ultimately recognize the rehabilitation movement type; for quality evaluation (also defined as evaluation of quality of lower-limb rehabilitation training), several parameters were selected and calculated using the processed data, and thereupon were used for the final scoring of the quality of lower-limb rehabilitation.

\section{Rehabilitation Motion Recognition}

Feature extraction

As observed in the conducted experiments, during the alternating weight-bearing exercise, the thighs of patients at Brunnstrom stage III have a stronger forward and synergistic movement pattern compared to patients at stage $\mathrm{V}$, which is in line with the lower limb mobility of both Brunnstrom stage III and V patients [17]. Based on experimental observations and previous research [15, 18], several features can be extracted from the filtered IMU sensors' data: maximum value, minimum value, peak-to-peak value, mean value, energy (ENE) accumulated in the XYZ axis and standard deviation (STD), among which the formula of ENE is given by:

$$
E N E=\sum_{i=1}^{n}\left(\left(x_{i}^{2}+y_{i}^{2}+z_{i}^{2}\right)^{\frac{1}{2}}-1\right)
$$

where $\left(x_{i}, y_{i}, z_{i}\right)$ represents the $\mathrm{X}, \mathrm{Y}, \mathrm{Z}$ components of a sample i of an IMU sensor.

To some extent, these features reflect the performance of the lower-limb movements of a patient. By way of illustration, the acceleration value is $1 \mathrm{~g}$ (maximum) or $0 \mathrm{~g}$ (minimum) when the lower leg is in the upright or horizontal state, respectively, thereby, the maximum and minimum values of the acceleration sensor reflect the extreme state of the lower limb, while the peak-to-peak values reflect the ROM of the limb. As a result, the relation between acceleration and lower-limb mobility can be treated as a mapping relation. The extracted features are shown in Table 6, where the count represents the total number of sub-features for each unique feature type. Each unique feature had six sub-features, i.e. X, Y, Z values from two IMU sensors. Raw samples represent the summation of the numbers of sub-features of all listed features except the Period (the time period can be calculated by the indicated sample rate, thus no need to be sampled).

\section{The CNN algorithm}

The CNN architecture employed in this paper is shown in Fig. 5. To protect against overfitting problems and evaluate the accuracy of the algorithm, K-fold cross-validation method was used. As shown in Table 6, the feature vector had 73 features in total. The input data were normalized before feeding it to the model, given as:

$$
X=\frac{X_{i}-X_{\min }}{X_{\max }-X_{\min }}
$$

where $X_{\max }$ is the maximum value of the vector, and $X_{\min }$ is the minimum value of the vector. A four-dimensional zero vector was padded ahead and behind the 
feature vector, thereby the original feature vector was transformed into a $9 \times 9$ matrix, which is convenient for multiplication with $2 \mathrm{D}$ CNN kernels. In the CNN architecture, the $9 \times 9$ matrix went through a series of convolution blocks and maxpool layers, before a SoftMax layer gave predictions for rehabilitation actions (see Fig. 5). A batch normalization layer and a Rectified linear unit (ReLU) activation function followed every convolution layer in a convolutional block [19].

\section{Quality Evaluation of Lower-limb Rehabilitation}

Quality evaluation parameters

According to the characteristics of stroke patients' movement, it is pivotal to select a reasonable evaluation index of rehabilitation movement, which can not only resolve the existing problems in qualitative evaluation, but also accurately determine the rehabilitation effect on patients. Previous studies have revealed that the lower-limb motion of stroke patients has the following characteristics compared to healthy people $[20,21]$ : (1) lower mean angular velocity of the extension and flexion for both hip joint and knee joint $(p<0.001)$; $(2)$ restricted range of extension of the hip joint $(p=0.001)$; (3) more intense limb trembling $(p<0.001)$. As per the points discussed above and the inputs from expert rehabilitation physicians, the following parameters were used for the lower-limb evaluation: range of motion (ROM) of joints, movement duration, the time difference between the beginning and the turning point (the point where the orientation of lower-limb movement alters between flexion and extension), the time difference between the turning point and the end of the movement, and the trembling of limbs on the plane of vertical motion, among which the limb trembling can be quantitatively assessed by introducing standard deviation. A weight was assigned to each parameter based on suggestions given by the rehabilitation physicians.

Generally, for the lower-limb evaluation of stroke patients, larger ROM of the joint indicates better lower-limb mobility, thus the ROM of the joint is positively contributed to the quality of rehabilitation. As mentioned above in this section, during lower limb movement, stroke patients usually tend to have lower angular velocity and a smaller range of motion than those of healthy subjects. Namely, they are arduous to keep a constant speed during joint movements, and the time duration from the beginning to the turning point of the movement and that from the turning point to the endpoint will be significantly different. Therefore, the time duration of the rehabilitation movement in one period is positively contributed to the quality of rehabilitation, while the time difference between joint extension and flexion is negatively contributed to the quality of rehabilitation. Furthermore, a larger mean square error of limb trembling on the plane of vertical motion along the Y-axis will reflect a larger deviation of motion, and it is negatively contributed to the quality of rehabilitation. Notably, there is a special case arising from the alternating weightbearing exercise, as both the hip joint and the knee joint are taken into account during this movement. In line with the situations of the other three movements, the ROM of hip joint is positively contributed to the quality of rehabilitation. Nevertheless, unlike the hip joint, the ROM of the knee joint during this movement is inversely proportional to the rehabilitation training quality, owing to the fact that a larger ROM of it reflects a higher extent of unwanted synergetic movement of the 
lower leg, indicating lower mobility of the lower limb. As discussed, the selected quality evaluation parameters of four rehabilitation movements are demonstrated in Table 7, with the assigned weights reflecting their respective contribution to the final score.

To obtain the values of the selected parameters, it is imperative to simplify the human skeleton in order to achieve the modeling and analysis of human movement, since it has an exceedingly complex structure. The essence of a simplified model is to map human body movement to human bone movement so that the human body can be reducible to points and straight lines [22]. In support of this, the simplified model proposed by Chen et al. [23], divides the human body into 17 parts and 14 points, representing the head, trunk, and limbs. For the rehabilitation movements selected in this paper, the limbs are fixed in the plane perpendicular to the face, and the positions of human body can be represented by lying flat and standing positions. Therefore, the human body can be simplified into the two schematic diagrams as shown in Fig. 6, where $\theta_{1}, \theta_{2}$ are the angles of a point on the $\mathrm{X}$-axis relative to the natural coordinate system at a certain point in the sequence in positions of lying flat, standing, respectively.

The ROM of the joints during motion can be calculated by the angle difference between the beginning point and the turning point of the movement, which is given by:

$$
\operatorname{ROM}(\theta)=\left|\tan ^{-1}\left(\frac{A_{x s}}{\sqrt{A_{y s}^{2}+A_{z s}^{2}}}\right)-\tan ^{-1}\left(\frac{A_{x t}}{\sqrt{A_{y t}^{2}+A_{z t}^{2}}}\right)\right|
$$

where $\operatorname{ROM}(\theta)$ is the range of motion (represented in angle value) of the joints during rehabilitation motion, $A_{x s}, A_{y s}$ and $A_{z s}$ are the corresponding acceleration values of $\mathrm{X}, \mathrm{Y}$ and $\mathrm{Z}$-axis sequences, respectively, at the beginning of the rehabilitation movement; $A_{x t}, A_{y t}$ and $A_{z t}$ are the corresponding acceleration values of $\mathrm{X}$, $\mathrm{Y}, \mathrm{Z}$-axis sequences, respectively, at the turning point of the movement.

The time duration in one period or the time difference between the extension and flexion of joints is given by:

$$
T=\left|\frac{N_{\text {start }} \pm N_{\text {end }}}{f}\right|
$$

where $\mathrm{f}$ is the sampling frequency; $N_{\text {start }}$ and $N_{\text {end }}$ is the number of sample points from the beginning to the turning point and from the turning point to the end of a rehabilitation movement, respectively; $T$ represents the time duration in one period when the sign in the equation is positive and represents the time difference between joint extension and flexion when the sign in the equation is negative.

The standard deviation of limb trembling on the plane of vertical motion along the $\mathrm{Y}$-axis is given by:

$$
S T D(y)=\sqrt{\frac{\left.\sum\left(\bar{y}-y_{i}\right)^{2}\right)}{N}}
$$

where $\operatorname{STD}(\mathrm{y})$ represents the standard deviation of trembling in the plane of vertical $\underline{\text { motion; }} \bar{y}$ represents the mean of the Y-axis sequence; $y_{i}$ represents the value of a 
point $\mathrm{i}$ (starts from 1 to the end of the data sequence) on the $\mathrm{Y}$-axis; $\mathrm{N}$ represents the number of sample points in the sequence.

Quality scoring system

To evaluate the quality of lower limb rehabilitation exercise of stroke patients, it is essential to compare a patient's rehabilitation movement data with that of healthy participants [24]. The bridge motion curves of a patient at Brunnstrom stage $\mathrm{V}$ and a healthy volunteer are shown in Fig. 7. The curves were down-sampled by taking one point at every 10 sample points, in which $\mathrm{Y}$-axis data sequences were processed using the mean removal to better compare the extents of lower-limb trembling on the plane of vertical motion. As can be clearly observed in Fig. 7, compared to the Brunnstrom stage $\mathrm{V}$ patient, the healthy participant possessed a better uniformity and periodicity in the motion curve, with a smoother pattern. In addition, there are significant differences in acceleration amplitude of motion curves between the healthy participant and the patient, which reveal the difference in mobility between them. For patients at a lower Brunnstrom stage, the difference in the aforementioned parameters compared to that of a healthy subject was more significant. Therefore, it can be said that the difference in evaluation parameters between patients and healthy participants is in line with that of their mobility. As a result, the evaluation parameters of the six healthy participants' rehabilitation training movements, were averaged and then taken as the template for further analysis. Similarly, for the other three rehabilitation movements, their templates were also attained by taking the mean values of the evaluation parameters of the six healthy volunteers. The score, which quantitatively reflects the mobility of a limb, can be obtained by:

$$
\text { Score }=\left(\sum \frac{\alpha_{i}}{\alpha_{s i}} \times W_{\alpha}+\sum \frac{\beta_{i}}{\beta_{s i}} \times W_{\beta}\right) \times 100 \%
$$

where "Score" refers to the gross score of a rehabilitation motion; $\alpha_{i}$ is a positive-contributed evaluation parameter (the subscript indicates the type); $\alpha_{s i}$ is the template corresponding to $\alpha_{i}$ (derived from the healthy participant); $\beta_{i}$ is a negative-contributed evaluation parameter; $\beta_{s i}$ is the template corresponding to $\beta_{i}$. A positive or negative-contributed evaluation parameter indicates a positive or negative contribution to the rehabilitation quality score. For instance, the ROM of a joint denoted as $\theta$ is considered as a type of $\alpha_{i}$ as it is positively contributed with rehabilitation quality; $W_{a}, W_{b}$ is the corresponding weight for parameter $\alpha_{i}, \beta_{i}$, respectively.

\section{Appendix}

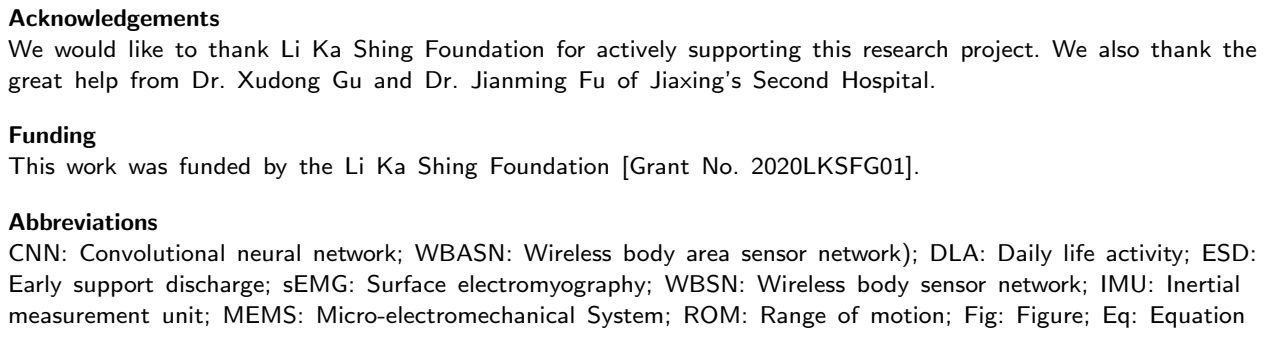


Availability of data and materials

The datasets used in this paper are available from the corresponding author on reasonable request.

Ethics approval and consent to participate

The experiment was approved by the ethics committee of the First Affiliated Hospital of Shantou University Medical College. The experimental procedure strictly complied with the Declaration of Helsinki.

Competing interests

The authors declare that they have no competing interests.

Consent for publication

Not applicable.

Authors' contributions

P. Ge: conducted the experiments, analyzed the experimental data, wrote the first draft of this manuscript. Z . Huang: conducted part of the verification experiment, made the statistical analysis, modified and edited the manuscript. G. Tang, A. Kumar and S. Mahmoud: conducted critical review and commenting. Q. Fang and Jian Chen: designed the experiment, supervised the research through the whole process, revised and finalized the manuscript. All authors read and approved the final manuscript.

Author details

${ }^{1}$ Department of Biomedical Engineering, Shantou University, Shantou, China. ${ }^{2}$ The First Affiliated Hospital of Shantou University Medical College, Shantou, China.

References

1. Dobkin, B.H.: Strategies for stroke rehabilitation. Lancet Neurology 3(9), 528-536 (2004). doi:10.1016/S1474-4422(04)00851-8

2. Dobkin, B.H.: A Rehabilitation-Internet-of-Things in the Home to Augment Motor Skills and Exercise Training. Neurorehabilitation and Neural Repair 31(3), 217-227 (2017). doi:10.1177/1545968316680490

3. Zhang, Jingjing; Li, Y.: Gait characteristics and rehabilitation strategies of stroke hemiplegia. Chinese Journal of Gerontology 39(05), 1044-1047 (2019)

4. Miller, K.K., Porter, R.E., DeBaun-Sprague, E., Van Puymbroeck, M., Schmid, A.A.: Exercise after stroke: Patient adherence and beliefs after discharge from rehabilitation. Topics in Stroke Rehabilitation 24(2), 1-7 (2016). doi:10.1080/10749357.2016.1200292

5. Brunnstrom, S.: Motor testing procedures in hemiplegia: Based on sequential recovery stages. American physical therapy association 46(4), 357-375 (1966)

6. Chen, W.-H., Yu, B., Xie, X.-H., Tu, X.-F.: Application and cost-effectiveness analysis of three-stage rehabilitation program in treating acute stroke. Chinese Journal of Clinical Rehabilitation 10(48), 31-33 (2006)

7. Langhorne, P., Taylor, G., Murray, G., Dennis, M., Anderson, C., Bautz-Holter, E., Dey, P., Indredavik, B., Mayo, N., Power, M., Rodgers, H., Ronning, O.M., Rudd, A., Suwanwela, N., Widen-Holmqvist, L., Wolfe, C.: Early supported discharge services for stroke patients: A meta-analysis of individual patients' data. Lancet 365(9458), 501-506 (2005). doi:10.1016/S0140-6736(05)70274-9

8. Patel, S., Park, H., Bonato, P., Chan, L., Rodgers, M.: A review of wearable sensors and systems with application in rehabilitation. Jornal of Neuroengineering and Rehabilitation 9(1), 1-17 (2012) doi:10.1186/1743-0003-9-21

9. Zhang, Z., Fang, Q., Gu, X.: Fuzzy inference system based automatic Brunnstrom stage classification for upper-extremity rehabilitation. Expert Systems with Applications 41(4 PART 2), 1973-1980 (2014). doi:10.1016/j.eswa.2013.08.094

10. Dobkin, B.H., Martinez, C.: Wearable Sensors to Monitor, Enable Feedback, and Measure Outcomes of Activity and Practice. Current Neurology and Neuroscience Reports 18(12) (2018). doi:10.1007/s11910-018-0896-5

11. Klaassen, B., van Beijnum, B.J.F., Held, J.P., Reenalda, J., van Meulen, F.B., Veltink, P.H., Hermens, H.J.: Usability Evaluations of a Wearable Inertial Sensing System and Quality of Movement Metrics for Stroke Survivors by Care Professionals. Frontiers in Bioengineering and Biotechnology 5(April), 1-12 (2017) doi:10.3389/fbioe.2017.00020

12. Liao, Y., Vakanski, A., Xian, M.: A deep learning framework for assessing physical rehabilitation exercises. IEEE Transactions on Neural Systems and Rehabilitation Engineering 28(2), 468-477 (2020). doi:10.1109/TNSRE.2020.2966249

13. Terui, Y., Suto, E., Konno, Y., Kubota, K., Iwakura, M., Satou, M., Nitta, S., Hasegawa, K., Satake, M., Shioya, T.: Evaluation of gait symmetry using a tri-axial accelerometer in stroke patients. NeuroRehabilitation 42(2), 173-180 (2018). doi:10.3233/NRE-172235

14. Wang, Y., Yu, L., Fu, J., Fang, Q.: Remote intelligent Brunnstrom assessment system for upper limb rehabilitation for post-stroke based on extreme learning machine. Journal of biomedical engineering 31(2), 251-256 (2014)

15. Hester, T., Hughes, R., Sherrill, D.M., Knorr, B., Akay, M., Stein, J., Bonato, P.: Using wearable sensors to measure motor abilities following stroke. In: International Workshop on Wearable and Implantable Body Sensor Networks (BSN'06), pp. 4-8 (2006). doi:10.1109/BSN.2006.57

16. Wang, Z., Zhang, D.: Progressive switching median filter for the removal of impulse noise from highly corrupted images. IEEE Transactions on Circuits and Systems II: Analog and Digital Signal Processing 46(1), 78-80 (1999). doi:10.1109/82.749102

17. Müller, G.: Movement therapy in hemiplegia. Beitrage zur Orthopadie und Traumatologie 24(4), 246-248 (1977) 
18. Patel, S., Dy, J.: Quantitative motor assessment in patients with mobility limiting conditions using wearable sensors. PhD thesis, Northeastern University (2012). doi:10.17760/d20002546

19. Le, C., Boser, Y., Denker, B., Henderson, D., Jackel, L.D.: Back-propagation applied to handwritten zip-code recognition (1992)

20. Wang, G.-m., Qi, R., Juan-tao, Y.: Biodynamic and kinematic characteristics of hemiplegic gait following stroke. Journal of Clinical Rehabilitative Tissue Engineering Research 11(40), 8169-8172 (2007)

21. Wan, Q., Wu, W., Liu, H.: Kinematic analysis of temporal-spatial parameters and lower extremity motions of hemiparetic stroke patients. Chinese Journal of Rehabilitation Medicine 29(11), 1026-1030 (2014). doi:10.3969/j.issn.1001-1242.2014.11.006

22. Sun, T., Li, H., Liu, Q., Duan, L., Li, M., Wang, C., Liu, Q., Li, W., Shang, W., Wu, Z., Wang, Y.: Inertial Sensor-Based Motion Analysis of Lower Limbs for Rehabilitation Treatments. Journal of Healthcare Engineering 2017 (2017). doi:10.1155/2017/1949170

23. Chen, Z., Lee, H.-J.: Knowledge-guided visual perception of 3-D human gait from a single image sequence. Systems, Man and Cybernetics, IEEE Transactions on 22, 336-342 (1992). doi:10.1109/21.148408

24. Houmanfar, R., Karg, M., Kulić, D.: Movement Analysis of Rehabilitation Exercises: Distance Metrics for Measuring Patient Progress. IEEE Systems Journal 10(3), 1014-1025 (2016). doi:10.1109/JSYST.2014.2327792

\section{Figures}

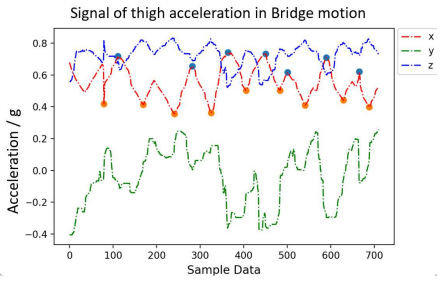

(a) Bridge motion

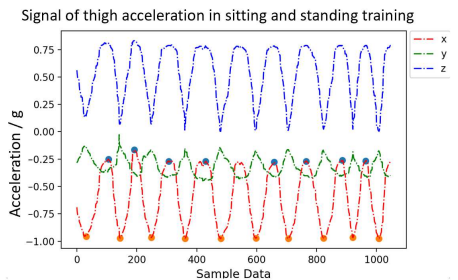

(b) Sitting and Standing Training

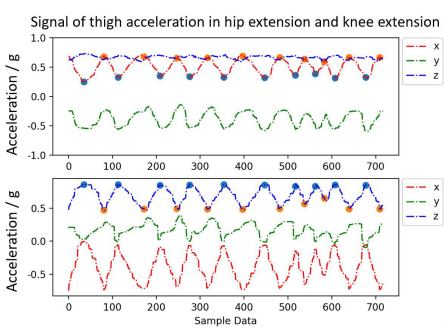

(c) Hip extension and knee extension

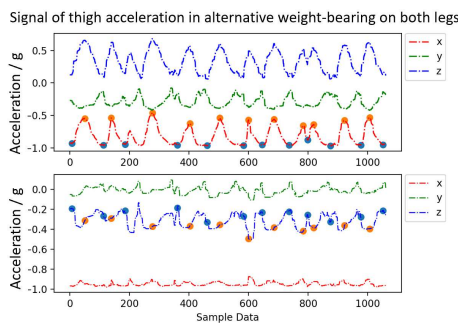

(d) Alternating weight-bearing on both limbs

Figure 1 Motion curve of lower limb rehabilitation training for a Brunnstrom stage III patient The red points are the turning points of the motion, and the blue points are the starting or ending points of the motion. The plus sign indicates that the movement direction is the same as the measurement direction of one axis of the sensor, while the minus sign indicates opposite movement against the IMU measurement direction of that axis.

Tables

Table 1 Training movement accuracy of the CNN-based algorithm obtained using the 5-fold cross-validation method.

\begin{tabular}{lllllll}
\hline Fold & Split (1) & Split (2) & Split (3) & Split (4) & Split (5) & Mean(Std) \\
\hline Accuracy (\%) & 100 & 100 & 92.3 & 92.3 & 91.7 & $95.3 \pm 3.9$ \\
\hline
\end{tabular}




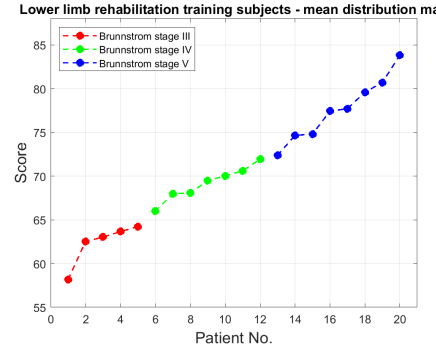

(a)

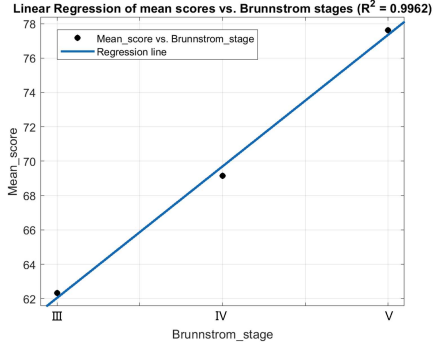

(b)

Figure 2 (a) The mean scores taken from the 4 selected rehabilitation motions of each patient. The colors represent the Brunnstrom stage classification evaluated by experienced physicians. (b) The linear regression of mean scores of each Brunnstrom stage shown in (a) versus Brunnstrom stages $\left(R^{2}=0.9962\right)$.
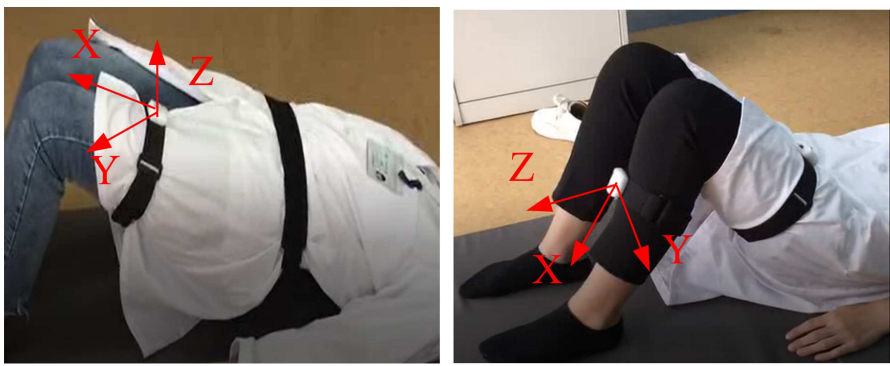

(a) IMU sensor placement during (b) IMU sensor placement during bridge motion during other rehabilitation motions

Figure 3 he IMU placements of four rehabilitation movements.

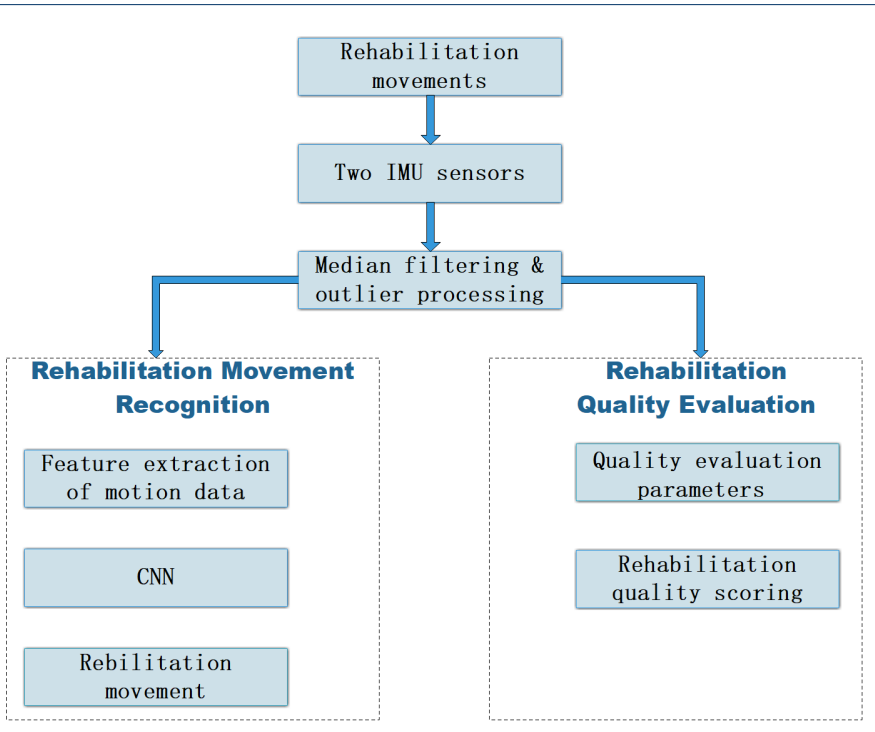

Figure 4 The flow chart of the proposed evaluation approach. 


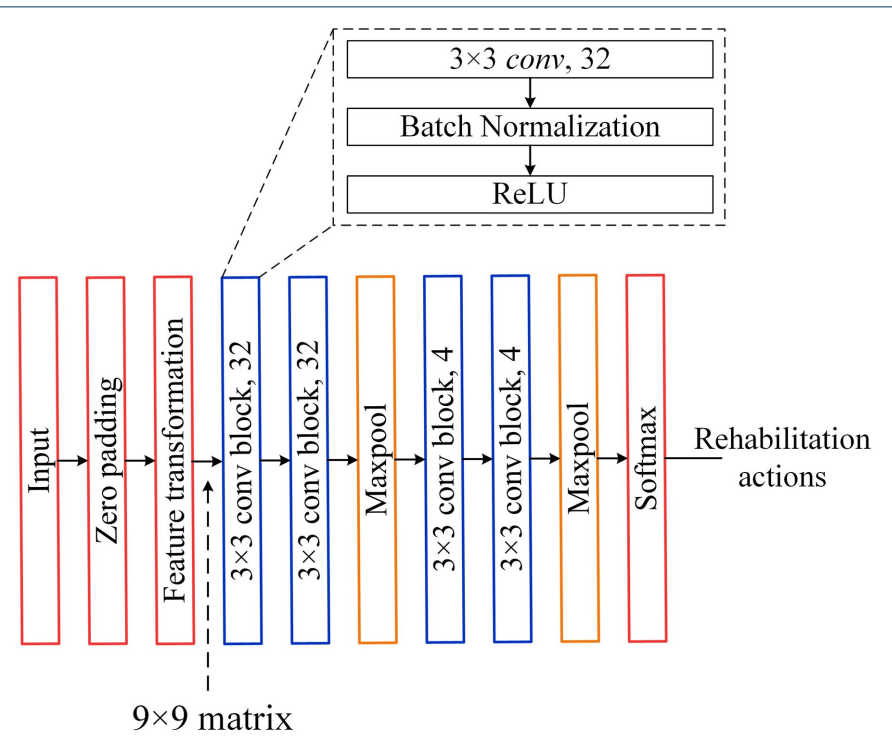

Figure 5 The schematic of the CNN architecture.

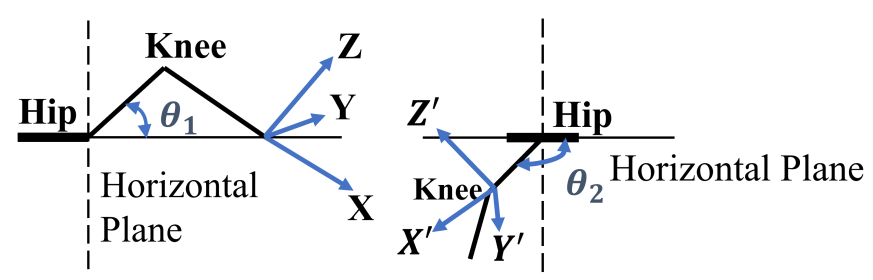

(a) Schematic diagram of ly- (b) Schematic diagram of standing ing flat

Figure 6 Simplified sketch of human joints.

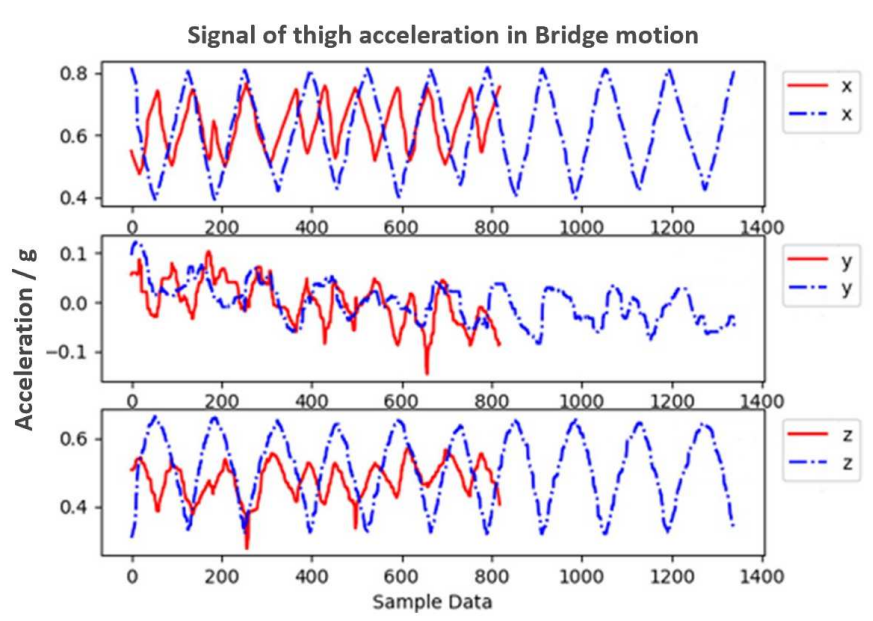

Figure 7 The curve of the bridge motion of a Brunnstrom stage $V$ patient (red) and a healthy volunteer (blue). The $\mathrm{X}$-axis represents the sampling data points. The $\mathrm{Y}$-axis represents the acceleration recorded for the motion relative to the gravitational acceleration $\mathrm{g}$ (the sign indicates the direction). 
Table 2 Calculations of four rehabilitation training scores for a Brunnstrom stage

\begin{tabular}{|c|c|c|c|c|c|c|c|}
\hline $\begin{array}{l}\text { Rehabilitation } \\
\text { exercises }\end{array}$ & $\begin{array}{l}\text { Processed } \\
\text { raw data }\end{array}$ & $\begin{array}{l}\text { Parameter } \\
\text { No. }\end{array}$ & Weight & $\begin{array}{l}\text { Equation No. } \\
\text { for parameters }\end{array}$ & Parametric & $\begin{array}{l}\text { Template } \\
\text { parameters }\end{array}$ & $\begin{array}{c}\text { Gross } \\
\text { score } \\
\text { (Eq. (6)) }\end{array}$ \\
\hline \multirow{4}{*}{ Bridge motion } & \multirow{4}{*}{$\begin{array}{c}\text { Thigh: } \\
\left(A_{x s}, A_{y s}, A_{z s}\right)^{1}=(0.43,0,0.80) \\
\left(A_{x h}, A_{y h}, A_{z h}\right)^{2}=(0.67,-0.11,0.66) \\
\text { N_start }{ }^{3}: 38.13 \\
\text { N_end }\end{array}$} & 1 & 2 & Eq. (3) & $\alpha_{1}: 16.8^{\circ}$ & $\alpha_{1 s}: 27.5^{\circ}$ & \multirow{4}{*}{61} \\
\hline & & 2 & 1 & Eq. $(4)^{5}$ : use "+" & $\alpha_{2}: 3.7 \mathrm{~s}$ & $\alpha_{2 s}: 6.1 \mathrm{~s}$ & \\
\hline & & 3 & 1 & Eq. (4): use"-" & $\beta_{1}: 0.11 \mathrm{~s}$ & $\beta_{1 s}: 0.11 \mathrm{~s}$ & \\
\hline & & 4 & 1 & Eq. (5) & $\beta_{2}: 0.18$ & $\beta_{2 s}: 0.04$ & \\
\hline \multirow{4}{*}{$\begin{array}{c}\text { Sitting } \\
\text { standing training }\end{array}$} & \multirow{4}{*}{ 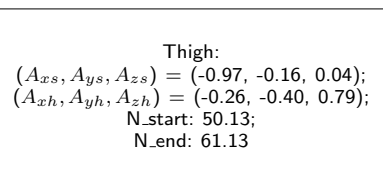 } & 1 & 1 & Eq. (3) & $\alpha_{1}: 64.0^{\circ}$ & $\alpha_{1 s}: 79.1^{\circ}$ & \multirow{4}{*}{61.6} \\
\hline & & 2 & 2 & Eq. (4): use "+" & $\alpha_{2}: 5.56 \mathrm{~s}$ & $\alpha_{2 s}: 9.52 \mathrm{~s}$ & \\
\hline & & 3 & 1 & Eq. (4): use" -" & $\beta_{1}: 0.55 \mathrm{~s}$ & $\beta_{1 s}: 0.30 \mathrm{~s}$ & \\
\hline & & 4 & 1 & Eq. (5) & $\beta_{2}: 0.09$ & $\beta_{2 s}: 0.05$ & \\
\hline \multirow{5}{*}{$\begin{array}{l}\text { Hip extension } \\
\text { knee extension }\end{array}$} & \multirow{5}{*}{ 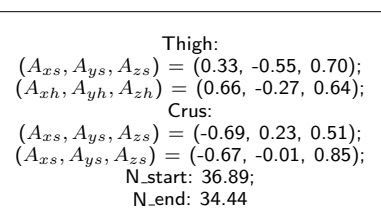 } & 1 & 1 & Eq. (3) & $\alpha_{1}: 23.2^{\circ}$ & $\alpha_{1 s}: 62.3^{\circ}$ & \multirow{5}{*}{68.7} \\
\hline & & 2 & 1 & Eq. (3) & $\alpha_{2}: 97.3^{\circ}$ & $\alpha_{2 s}: 108.9^{\circ}$ & \\
\hline & & 3 & 1 & Eq. (4): use "+" & $\alpha_{3}: 3.6 \mathrm{~s}$ & $\alpha_{3 s}: 6.32 \mathrm{~s}$ & \\
\hline & & 4 & 1 & Eq. (4): use"-" & $\beta_{1}: 0.12 \mathrm{~s}$ & $\beta_{1 s}: 0.18 \mathrm{~s}$ & \\
\hline & & 5 & 1 & Eq. (5) & $\beta_{2}: 0.10$ & $\beta_{2 s}: 0.06$ & \\
\hline \multirow{5}{*}{$\begin{array}{c}\text { Alternating weight-bearing } \\
\text { on both legs }\end{array}$} & \multirow{5}{*}{$\begin{aligned} & \text { Thigh: } \\
&\left(A_{x s}, A_{y s}, A_{z s}\right)=(-0.96,-0.17,0.10) \\
&\left(A_{x h}, A_{y h}, A_{z h}\right)=(-0.56,-0.39,0.60) \\
& \text { Crus: } \\
&\left(A_{x s}, A_{y s}, A_{z s}\right)=(-0.96,0.01,-0.25) \\
&\left(A_{x h}, A_{y h}, A_{z h}\right)=(-0.92,-0.02,-0.38) \\
& \text { N_start: } 69.56 \\
& \text { N_end: } 38.22\end{aligned}$} & 1 & 2 & Eq. (3) & $\alpha_{1}: 39.8^{\circ}$ & $\alpha_{1 s}: 87.5^{\circ}$ & \multirow{5}{*}{58.8} \\
\hline & & 2 & 1 & Eq. (4): use "+" & $\alpha_{2}: 5.4 \mathrm{~s}$ & $\alpha_{2 s}: 7.2 \mathrm{~s}$ & \\
\hline & & 3 & 1 & Eq. (3) & $\beta_{1}: 7.4^{\circ}$ & $\beta_{1 s}: 4.2^{\circ}$ & \\
\hline & & 4 & 1 & Eq. (4): use"-" & $\beta_{2}: 1.57 \mathrm{~s}$ & $\beta_{2 s}: 0.48 \mathrm{~s}$ & \\
\hline & & 5 & 1 & Eq. (5) & $\beta_{3}: 0.04$ & $\beta_{3 s}: 0.07$ & \\
\hline \multicolumn{8}{|c|}{$\begin{array}{l}\text { Notes: } \\
\text { 1. } A_{x s}, A_{y s}, A_{z s} \text { refer to mean values of starting/ending point of thigh/crus sensor. } \\
\text { 2. } A_{x h}, A_{y h}, A_{z h} \text { refer to mean values of turning point of thigh/crus sensor. } \\
\text { 3. N_start refers to average sequence length from starting point to turning point. } \\
\text { 4. N_end refers to average sequence length from turning point to end point. } \\
\text { 5. In Equation No. }(4), \mathrm{f}=20 \text {. }\end{array}$} \\
\hline
\end{tabular}

Table 3 Mean and standard deviation of scores of each of the rehabilitation motions of patients at Brunnstrom stage III, IV, V.

\begin{tabular}{|c|c|c|c|c|}
\hline Rehabilitation exercises & $\begin{array}{c}\text { Brunnstrom } \\
\text { III }\end{array}$ & $\begin{array}{c}\text { Brunnstrom } \\
\text { IV }\end{array}$ & $\begin{array}{c}\text { Brunnstrom } \\
\vee\end{array}$ & $\begin{array}{c}\text { Spearman's rank-order } \\
\text { correlation }\end{array}$ \\
\hline Bridge motion & $59.7 \pm 1.8$ & $65.7 \pm 6.0$ & $81.2 \pm 6.2$ & \multirow{4}{*}{$\begin{array}{c}r_{s}(18)=0.847 \\
\mathrm{p} 0.001 \\
r_{s}(18)=0.837 \\
\mathrm{p} 0.001 \\
r_{s}(18)=0.746 \\
\mathrm{p} 0.001 \\
r_{s}(18)=0.558 \\
\mathrm{p}=0.011\end{array}$} \\
\hline Sitting Standing Training & $63.8 \pm 5.4$ & $69.6 \pm 3.0$ & $78.4 \pm 5.1$ & \\
\hline $\begin{array}{c}\text { Hip extension } \\
\& \text { knee extension }\end{array}$ & $56.7 \pm 4.2$ & $67.2 \pm 3.0$ & $70.5 \pm 4.6$ & \\
\hline $\begin{array}{c}\text { Alternating weight-bearing } \\
\text { on both legs }\end{array}$ & $69.0 \pm 4.5$ & $74.2 \pm 5.2$ & $80.4 \pm 8.1$ & \\
\hline
\end{tabular}

Table 4 Four lower-limb rehabilitation exercises used in the experiment.

\begin{tabular}{|c|c|c|}
\hline Name of rehabilitation exercises & Related motor muscles & $\begin{array}{l}\text { Objective of rehabilitation } \\
\text { exercises }\end{array}$ \\
\hline Bridge motion & $\begin{array}{l}\text { Gluteus maximus, } \\
\text { quadriceps femoris, } \\
\text { gluteus medius } \\
\text { iliopsoas }\end{array}$ & $\begin{array}{l}\text { Increased pelvic control im- } \\
\text { proved selective leg muscle } \\
\text { extension }\end{array}$ \\
\hline Sitting and Standing Training & $\begin{array}{l}\text { Iliopsoas } \\
\text { gluteus } \\
\text { maximus }\end{array}$ & $\begin{array}{l}\text { Increased control of lower } \\
\text { limbs }\end{array}$ \\
\hline Hip extension and knee extension & $\begin{array}{c}\text { Gluteus maximus } \\
\text { biceps femoris } \\
\text { iliopsoas }\end{array}$ & Inhibit lower limb spasm \\
\hline Alternating weight-bearing on both limbs & $\begin{array}{l}\text { Iliopsoas } \\
\text { quadriceps femoris } \\
\text { gluteus medius } \\
\text { gluteus maximus } \\
\text { triceps calf } \\
\text { tibialis anterior }\end{array}$ & $\begin{array}{l}\text { Experience } \\
\text { weight-bearing on upper } \\
\text { and lower limbs to prepare } \\
\text { for walking }\end{array}$ \\
\hline
\end{tabular}


Table 5 General information of participants.

\begin{tabular}{ll}
\hline Information type & Information \\
\hline Male/ Female & $17 / 9$ \\
Age (mean \pm standard deviation) & $62 \pm 11.6$ \\
Left/Right Hemiplegia & $10 / 11$ \\
Brunnstrom III/IV/V/VI & $5 / 7 / 8 / 6$ \\
Onset time (mean \pm standard deviation) (month) & $8.9 \pm 7.2$ \\
\hline
\end{tabular}

Table 6 Feature list of motion acceleration curves of rehabilitation movements of lower limbs.

\begin{tabular}{|c|c|c|}
\hline Feature & Count & Description \\
\hline Max \& min & $2 \times 6$ & Lower extremity rehabilitation exercise limit positions \\
\hline Peak-to-peak & $1 \times 6$ & The range of motion of lower limb rehabilitation movement \\
\hline Mean & $1 \times 6$ & $\begin{array}{l}\text { The mean variation in limb posture during lower limb rehabilitation } \\
\text { movement }\end{array}$ \\
\hline Std & $1 \times 6$ & $\begin{array}{l}\text { The fluctuation degree in limb posture during lower limb rehabilitation } \\
\text { movement }\end{array}$ \\
\hline ENE & $1 \times 6$ & Energy accumulated in the $X Y Z$ axis during a rehabilitation exercise \\
\hline Period & & The time required to complete a rehabilitation exercise \\
\hline Raw samples & $1 \times 36$ & Rehabilitation motion sequence description \\
\hline
\end{tabular}

Table 7 Quality evaluation form of lower limb rehabilitation movements.

\begin{tabular}{llc}
\hline Rehabilitation exercises & Evaluation parameters & Weight \\
\hline \multirow{4}{*}{ Bridge motion } & 1. ROM of hip joint & +2 \\
& 2. Period of motion & +1 \\
& 3. Time difference between hip extension and flexion & -1 \\
& 4. Standard deviation of Y-axis of thigh & -1 \\
\hline \multirow{5}{*}{ Sitting and Standing Training } & 1. ROM of knee joint & +1 \\
& 2. Period of motion & +2 \\
& 3. Time difference between knee extension and flexion & -1 \\
& 4. Standard deviation of Y-axis of thigh & -1 \\
\hline & 1. ROM of hip joint & +1 \\
& 2. ROM of knee joint & +1 \\
Hip extension and knee extension & 3. Period of motion & +1 \\
& 4. Time difference between knee extension and knee bend & -1 \\
& 5. Standard deviation of Y-axis of thigh & -1 \\
\hline & 1. ROM of hip joint & +2 \\
Alternating weight-bearing & 2. Period of motion & +1 \\
on both legs & 3. ROM of knee joint & -1 \\
& 4. Time difference between knee extension and knee bend & -1 \\
Notes: & 5. Standard deviation of Y-axis of thigh & -1 \\
\hline The plus sign (+) indicates the parameter is positively contributed to the quality score; & \\
\hline
\end{tabular}

